\title{
Assessment of cardiovascular risk in primary health care
}

\author{
PÄIVI KORHONEN ${ }^{1,2}$, RISTO VESALAINEN ${ }^{3,4}$, PERTTI AARNIO 5 , \\ HANNU KAUTIAINEN ${ }^{6,7}$, SALME JÄRVENPÄÄ ${ }^{8} \&$ ILKKA KANTOLA $^{3}$
}

${ }^{1}$ Central Satakunta Health Federation of Municipalities, Harjavalta, Finland, ${ }^{2}$ Institute of Clinical Medicine, Family Medicine, University of Turku, Turku, Finland, ${ }^{3}$ Department of Medicine, University of Turku, Turku, Finland, ${ }^{4}$ Pulssi Medical Center, Turku, Finland, ${ }^{5}$ Department of Surgery, Satakunta Hospital District, Pori, Finland, ${ }^{6}$ Department of Family Practice, Central Finland Central Hospital, Fyväskylä, Finland, ${ }^{7}$ Unit of Primary Health Care, Kuopio University Hospital, Kuopio, Finland, and ${ }^{8}$ Medcare Foundation, Ä̈̈nekoski, Finland

\begin{abstract}
Objective. This study aimed at investigating whether cardiovascular risk factors and their impact on total risk estimation differ between men and women. Design. Cross-sectional cohort study. Subjects. Finnish cardiovascular risk subjects ( $\mathrm{n}=904)$ without established cardiovascular disease, renal disease, or known diabetes. Main outcome measures. Ankle-brachial index (ABI), estimated glomerular filtration rate (eGFR), oral glucose tolerance test, and total cardiovascular risk using SCORE risk charts. Results. According to the SCORE risk charts, 27.0\% (95\% CI 23.1-31.2) of the women and $63.1 \%$ (95\% CI 58.3-67.7) of the men $(\mathrm{p}<0.001)$ were classified as high-risk subjects. Of the women classified as low-risk subjects according to SCORE, $25 \%$ had either subclinical peripheral arterial disease or renal insufficiency. Conclusions. The SCORE system does not take into account cardiovascular risk factors typical in women, and thus underestimates their total cardiovascular risk. Measurement of ABI and eGFR in primary care might improve cardiovascular risk assessment. especially in women.
\end{abstract}

Key Words: Ankle-brachial index, cardiovascular risk estimation, gender difference, glucose disorders, renal function

\section{Introduction}

Cardiovascular disease (CVD) is the main cause of death in Europe and accounts for 54\% of deaths in women and $43 \%$ of deaths in men [1]. With the ageing of the European population and with elderly women outnumbering elderly men, the gender gap in CVD mortality continues to widen.

The SCORE (Systematic COronary Risk Evaluation) risk charts [2], which are recommended to estimate the risk of dying of CVD over the next decade, show this gender gap clearly: a 60 -year-old woman has an almost identical risk to a 50-year-old man. Therefore, it is recommended that in women total CVD risk should be estimated to 70 years instead of 60 years, both the absolute risk and relative risk should be estimated, and risk factors that are particularly important for women, i.e. diabetes and obesity, should be taken into account [3].
Primary prevention efforts of CVD are less simple to implement than secondary prevention efforts, where an array of evidence-based therapies is available. In primary prevention, high-risk strategies focus on the detection and treatment of individuals identified as being at unacceptably high risk of CVD [2]. The key question to be resolved is how to best define high risk; to use total CVD risk-estimating tools like SCORE, to assess additional risk factors like renal function and glucose homeostasis, or to use indicators of asymptomatic atherosclerosis like measurement of ankle-brachial index (ABI). We applied these methods, which are easily available to a general practitioner (GP), to compare CVD risk estimation between male and female subjects. Our study subjects were selected from the general population by using a two-stage screening method, in order to create a cohort of subjects who form a typical dilemma

Correspondence: Päivi Korhonen, MD PhD, Central Satakunta Health Federation of Municipalities, Jokikatu 3, 29200 Harjavalta, Finland. Tel: +358-40-7653257. Fax: +358-2-6741180. E-mail: paivi.e.korhonen@fimnet.fi 
More women than men die from cardiovascular disease in Europe, but the non-conventional risk factors in women may remain undiagnosed or ignored.

- In a cohort of middle-aged cardiovascular risk subjects in primary care, $27 \%$ of the women and $63 \%$ of the men $(\mathrm{p}<0.001)$ were classified as high-risk subjects according to the SCORE risk charts.

- Of the women classified as low-risk subjects according to SCORE, $25 \%$ had either subclinical peripheral arterial disease or renal insufficiency.

- Measurement of ABI and eGFR in primary care might improve cardiovascular risk assessment, especially in women.

for a GP: Which patients should receive intensive primary prevention therapy?

\section{Material and methods}

\section{Subjects}

The study population was drawn from the Harmonica Project, a population survey designed to evaluate cardiovascular risk factors in people aged 45 to 70 years living in two communities, Harjavalta and Kokemäki, in south-western Finland. A two-stage screening strategy was used: a risk factor survey was mailed to 6013 inhabitants, and of the 4450 (74\%) subjects willing to participate in the project, those having at least one cardiovascular risk factor $(\mathrm{n}=2752)$ were invited to an enrolment examination performed by a trained nurse. High-risk subjects $(\mathrm{n}=1928)$ with hypertension, metabolic syndrome as defined by the International Diabetes Federation criteria [4], impaired glucose homeostasis as defined by the WHO criteria [5], body mass index $\geq 30 \mathrm{~kg} / \mathrm{m}^{2}$, or a 10 -year risk of cardiovascular disease death of $5 \%$ or more according to the SCORE system [3] were further examined by a physician. In Harjavalta, ankle-brachial index (ABI) was measured in 972 of 1076 (90\%) non-claudicant risk subjects who did not have established CVD, renal disease, or previously known diabetes. A detailed description of the enrolment and examination methods has been published earlier [6]. In this paper, we analyse the test results of 904 study subjects who had valid measurements of ABI, OGTT, and renal function.

Estimation of renal function, subclinical arterial disease, and glucose homeostasis

Renal function was measured with plasma creatinine level (enzymatic method, Olympus ${ }^{\circledR}$ AU640, Japan) and estimated glomerular filtration rate (eGFR) calculated by the modified four-variable MDRD Study equation [7] $175 \times\left(\mathrm{P}_{\mathrm{Cr}} / 88.4\right)^{-1.154} \mathrm{x}(\text { Age })^{-0.203} \mathrm{x}$ (0.742 if female) $\times$ (1.21 if black), where $\mathrm{P}_{\mathrm{Cr}}=$ plasma creatinine in $\mu \mathrm{mol} / \mathrm{l}$, and age is expressed in years. Race was not applicable in our study because all patients were white. Because the MDRD formula is based on data from patients with advanced renal failure, the results may not be valid in subjects with normal or near normal glomerular filtration rates. Therefore we report only eGFR levels $<60 \mathrm{~mL} /$ $\mathrm{min} / 1.73 \mathrm{~m}^{2}$ as renal dysfunction.

ABI was determined from blood pressure measurements in the arms and ankles with the patient supine. Systolic blood pressure (SBP) in the brachial artery was measured in both arms using a blood pressure cuff and Doppler instrument (UltraTec ${ }^{\circledR}$ PD1v with a vascular probe of $5 \mathrm{MHz}$, United Kingdom) in the antecubital fossa. SBP at the left and right dorsalis pedis arteries, and if not found at the left and right posterior tibial arteries, was then measured with Doppler detection with a blood pressure cuff applied to the ankle just proximal to the malleoli. ABI was the lower ankle systolic blood pressure divided by the higher brachial systolic blood pressure. Using the lower of the ankle pressures has been shown to identify a higher number of patients with increased risk of future cardiovascular events than using the higher ankle pressure in calculating $\mathrm{ABI}$ [8]. ABI value $<1.00$ was used to indicate subclinical peripheral arterial disease.

OGTT was performed by measuring a fasting plasma glucose and a two-hour plasma glucose after ingestion of a glucose load of $75 \mathrm{~g}$ anhydrous glucose dissolved in water. Glucose values were measured from capillary whole blood with $\mathrm{HemoCue}{ }^{\circledR}$ Glucose $201+$ system (Ängelholm, Sweden).

\section{Informed consent}

All of the participants provided written informed consent for the project and subsequent medical research. The study protocol and consent forms were reviewed and approved by the ethics committee of Satakunta Hospital District.

\section{Statistical analysis}

The data are presented as the means with standard deviations (SD) or as counts with percentages. Statistical comparison between genders in was made by t-test, Mann-Whitney test, or chi-squared test. Confidence interval for the ratio between genders was estimated using bias-corrected bootstrapping. In order to make comparisons with SCORE values, ABI values and eGFR values were divided into sex-specific 
tertiles: ABI women: 0.65-1.03, 1.04-1.12, 1.13-1.39; ABI men: $0.60-1.04,1.05-1.14,1.15-1.39$; eGFR women: 35-70, 70-81, 82-172; eGFR men: 48-77, 78-90, 90-197.

\section{Results}

We evaluated 481 women and 423 men aged 45 to 70 years (mean age $59 \pm 7$ years) with hypertension, metabolic syndrome, newly detected glucose disorders, BMI of at least $30 \mathrm{~kg} / \mathrm{m}^{2}$, or SCORE risk of at least $5 \%$. None of the study subjects had clinical cardiovascular or renal disease or known diabetes.

Table I shows the demographic and clinical characteristics of the study subjects according to gender. With regard to the cardiovascular risk factors entered into the SCORE model, men smoked more often than women, but women had higher total cholesterol values. Of the cardiovascular risk factors not included in the SCORE model, women tended to have higher body mass index, higher fasting and two-hour glucose values, as well as lower eGFR than men.

According to SCORE risk charts, $130 / 481(27.0 \%$ [95\% CI 23.1-31.2]) of the women and 267/423 (63.1\% [95\% CI 58.3-67.7]) of the men $(\mathrm{p}<0.001)$ were classified as high-risk subjects (SCORE $\geq 5 \%$ ) when extrapolated to 60 years. Ratio between male and female in SCORE value was 1.90 (95\% CI 1.64$2.20), p<0.001$. When the risks of women and men were extrapolated to 70 years using SCORE risk charts, 412/481 (85.7\% [95\% CI 82.2-88.7]) and $423 / 423$ (100.0\% [95\% CI 99.1-100)] had SCORE $\geq 5 \%$, respectively.

SCORE values were significantly higher in men across all tertiles of ABI and eGFR (Figures 1A and B), and across all stages of glucose homeostasis (Figure 1C). When the risks of the diabetic subjects were multiplied by four in women and by two in men as recommended by the SCORE project group [2], 10 -year risk of cardiovascular death was comparable between genders.

According to the MDRD formula, the crude prevalence of renal insufficiency defined as eGFR $<60 \mathrm{~mL} / \mathrm{min} / 1.73 \mathrm{~m}^{2}$ was $51 / 481$ (10.6\% [95\% CI 8.0-13.7]) in women and $16 / 423(3.8 \%$ [95\% CI $2.2-16.1])$ in men $(p<0.001)$. Of the patients with renal insufficiency, 30/51 (58.8\%) female and 3/16 $(18.6 \%)$ male subjects had SCORE $<5 \%$ when extrapolated to 60 years.

Of the 51 female patients with eGFR $<60 \mathrm{~mL} /$ $\mathrm{min} / 1.73 \mathrm{~m}^{2}$, only three $(5.9 \%)$ had elevated plasma creatinine concentration, i.e. $\geq 107 \mu \mathrm{mol} / \mathrm{L}$ as defined by European guidelines [9]. Respectively, nine male patients of the $16(56.3 \%)$ with renal insufficiency had plasma creatinine $\geq 115 \mu \mathrm{mol} / \mathrm{L}$.

Table I. Characteristics of the subjects according to gender.

\begin{tabular}{lccr}
\hline & Female $\mathrm{n}=481$ & Male $\mathrm{n}=423$ & P-value \\
\hline Demographic: & & & \\
Age, years, mean (SD) & $59(7)$ & $58(7)$ & 0.056 \\
Body mass index, $\mathrm{kg} / \mathrm{m}^{2}$, mean (SD) & $29.7(5.5)$ & $28.6(4.1)$ & 0.004 \\
Waist circumference, cm, mean (SD) & $93.5(12.3)$ & $101.3(10.6)$ & \\
Current smokers, $\mathrm{n}(\%)$ & $58(12)$ & $95(22)$ & $<0.001$ \\
Clinical: & & & \\
Blood pressure, mmHg, mean (SD) & & & \\
$\quad$ Systolic & $149.0(17.7)$ & $148.4(17.2)$ & 0.625 \\
$\quad$ Diastolic & $88.1(8.0)$ & $89.6(8.6)$ & 0.007 \\
$\quad$ Pulse pressure & $60.9(14.2)$ & $58.8(13.1)$ & 0.023 \\
$\quad$ Ankle-brachial index, mean (SD) & $1.08(0.11)$ & $1.09(0.12)$ & 0.216 \\
$\quad$ eGFR, mL/min/1.73 m ${ }^{2}$, mean (SD) & $77.1(15.4)$ & $85.1(16.8)$ & $<0.001$ \\
SCORE, \%, mean (SD) & $4(6)$ & $8(6)$ & \\
Biochemical: & & & \\
Total cholesterol, mmol/L, mean (SD) & $5.36(0.96)$ & $5.22(0.90)$ & 0.034 \\
LDL cholesterol, mmol/L, mean (SD) & $3.20(0.87)$ & $3.18(0.81)$ & 0.780 \\
HDL cholesterol, mmol/L, mean (SD) & $1.56(0.39)$ & $1.39(0.39)$ & $<0.001$ \\
Triglycerides, mmol/L, mean (SD) & $1.35(0.61)$ & $1.47(0.75)$ & 0.220 \\
Fasting glucose, mmol/L, mean (SD) & $5.59(1.14)$ & $5.77(1.01)$ & $<0.001$ \\
Two-hour glucose, mmol/L, mean (SD) & $7,81(2.54)$ & $7.51(2.43)$ & 0.026 \\
Current medication, (\%): & & & \\
Vasodilatators & $133(28)$ & $128(30)$ & 0.363 \\
Beta-blockers & $112(23)$ & $81(19)$ & 0.098 \\
Diuretics & $53(11)$ & $39(9)$ & 0.341 \\
Statins & $76(16)$ & $57(14)$ & 0.273 \\
\hline
\end{tabular}

Note: eGFR = estimated glomerular filtration rate; SCORE $=$ Systematic COronary Risk Evaluation; $\mathrm{LDL}=$ low-density cholesterol; HDL $=$ high-density cholesterol. 

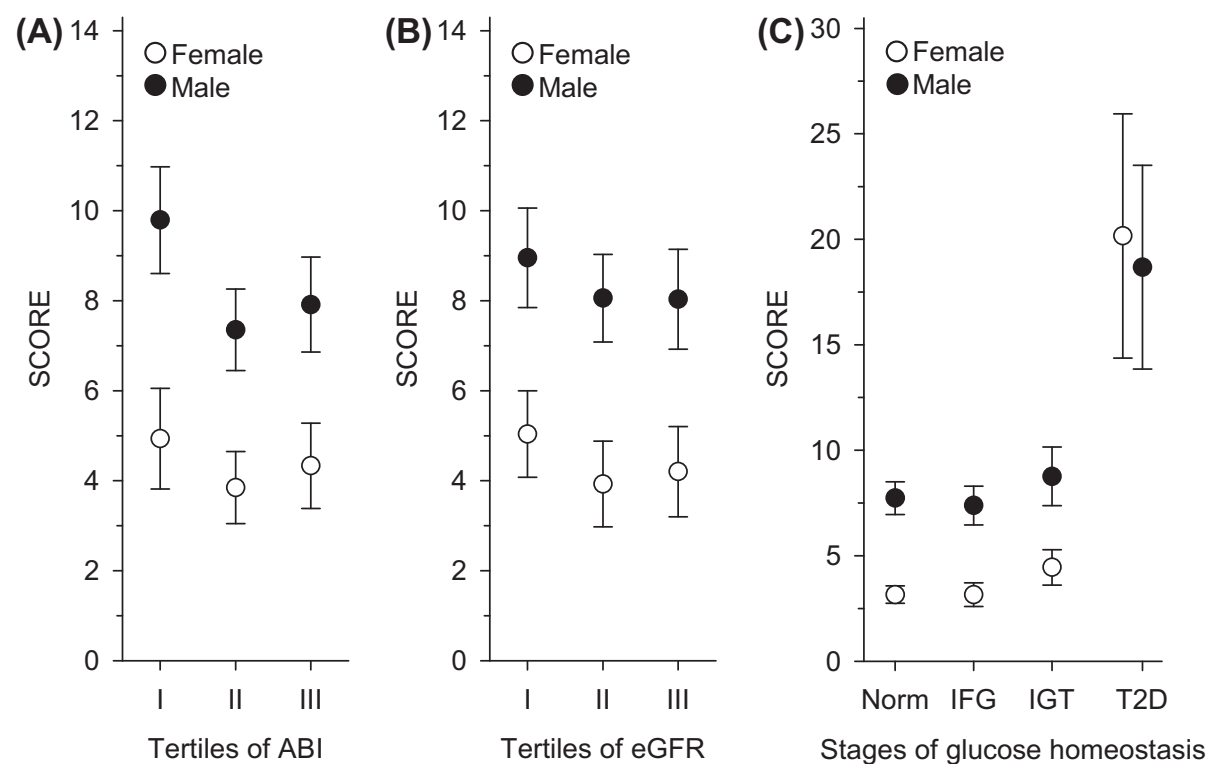

Figure 1. SCORE values across tertiles of ABI (A), eGFR (B), and stages of glucose homeostasis (C). Note: SCORE = Systematic COronary Risk Evaluation; $\mathrm{ABI}=$ ankle-brachial index; eGFR = estimated glomerular filtration rate; $\mathrm{IFG}=$ impaired fasting glucose; IGT $=$ impaired glucose tolerance; $\mathrm{T} 2 \mathrm{D}=$ type 2 diabetes.

The prevalence of subclinical peripheral arterial disease defined as $\mathrm{ABI}<1.00$ was $96 / 481(20.0 \%$ [95\% CI 16.5-23.8]) in women and 75/423 (17.8\% [95\% CI 14.2-21.7]) in men $(\mathrm{p}=0.393)$. Of the 96 women with low ABI, 57 (59.4\%) had SCORE $<5 \%$ when extrapolated to 60 years. The corresponding figures in men were $7 / 75$ (9.3\%).

Of the glucose disorders, the prevalence of impaired glucose tolerance (IGT) was 89/481 (18.5\% [95\% CI 15.1-22.3]) in women and $67 / 423(15.8 \%$ [95\% CI 12.5-19.7]) in men $(p=0.29)$, and the prevalence of previously undetected type 2 diabetes was $28 / 481(5.8 \%$ [95\% CI 3.9-8.3]) in women and $22 / 423(5.2 \%$ [95\% CI 3.3-7.8]) in men $(\mathrm{p}=0.68)$. Of the subjects with IGT, 58/156 (37.2\%) had SCORE $<5 \%$ when extrapolated to 60 years, and $54 / 58(93.1 \%)$ of them were women.

\section{Discussion}

Our results from a cohort of 904 cardiovascular risk subjects show that the SCORE system does not take into account typical cardiovascular risk factors in women. Of the major risk factors incorporated in the SCORE system, only total cholesterol values were higher in women than in men, but the difference is explained by the higher high-density lipoprotein cholesterol concentration in women. The lack of major risk factors may at least partly explain the results of the Euro Heart Survey [10], indicating that women with stable coronary heart disease are less likely to be referred to testing for ischaemia, less likely to be referred for revascularization, and less likely to receive secondary preventive therapies. At the same time, the non-conventional risk factors may remain undiagnosed or ignored, leading to the high cardiovascular mortality rates in women.

The causes of cardiovascular risk factors may alter between genders; for example sedentary lifestyle and smoking are associated with left ventricular diastolic dysfunction only in women [11]. It has also been suggested that clinical manifestations of CVD in women may be different from those commonly observed in males [3]. In our study, measurement of ABI revealed subclinical peripheral arterial disease (PAD) in every fifth of the study subjects - evenly in women and in men. This is in concordance with other studies showing that the prevalence of PAD does not vary with gender [12,13]. Recent metaanalysis of 16 cohort studies, in which participants aged 47 to 78 years were derived from a general population, showed that in women with ABI 0.911.00 the hazard ratio for cardiovascular mortality was 1.84 (95\% CI 1.53-2.22) and in men 1.68 (95\% CI 1.40-2.00) compared with reference ABI 1.11-1.20 [14]. In our study, out of 351 female subjects classified into the low-risk category according to the SCORE system, measurement of ABI reclassified 57 $(16 \%)$ into the high-risk category needing intensive lifestyle counselling, possibly preventive medication, and certainly indicating follow-up. In contrast to the other cardiovascular diseases, PAD is easily detectable with determination of ABI. ABI level $\leq 0.90$ is $95 \%$ sensitive in detecting angiogram-positive $\mathrm{PAD}$ and almost $100 \%$ specific in identifying apparently healthy individuals [15]. Variability of ABI measurement is comparable with that of routine blood pressure [16]. PAD is rarely an isolated disease of the lower limb 
arteries; patients with PAD frequently also have coronary heart disease or cerebrovascular disease [17-19]. These facts also strengthen the role of ABI in cardiovascular risk estimation in primary care.

In our study, the prevalence of chronic kidney disease defined as eGFR $<60 \mathrm{~mL} / \mathrm{min} / 1.73 \mathrm{~m}^{2}$ according to the MDRD formula was three times higher in women than in men. Chronic kidney disease is an independent risk factor for cardiovascular diseases; individuals with GFR $<60 \mathrm{ml} / \mathrm{min} / 1.73 \mathrm{~m}^{2}$ have an approximate $16 \%$ increase, and individuals with GFR $<30 \mathrm{ml} / \mathrm{min} / 1.73 \mathrm{~m}^{2}$, a $30 \%$ increase in cardiovascular mortality [20]. If we would had estimated renal function on the basis of plasma creatinine alone, we would have missed $80 \%$ of the patients with renal insufficiency.

In contrast to $\mathrm{ABI}<1.00$ and eGFR $<60 \mathrm{~mL} /$ $\mathrm{min} / 1.73 \mathrm{~m}^{2}$, there is no compelling evidence to suggest that cardiovascular risk associated with IGT is substantial [21]. Thus, the chief advantage of conducting an oral glucose tolerance test is to identify people with undiagnosed type 2 diabetes, who encompassed only $5 \%$ of our study population.

European guidelines recommend that SCORE risk should be extrapolated to a higher degree in women than in men, i.e. 70 years instead of 60 years [2]. Having performed this in our study cohort of women at risk of CVD, $86 \%$ of the women without previously known CVD or diabetes or renal disease would have been labelled as high-risk patients (SCORE risk $\geq 5 \%$ ) needing intensive risk management. The same phenomenon was observed in Norway; nearly all women aged 65 years and older were classified as at "high risk" [22]. In Sweden, the SCORE guidelines were applied to a large population-based cohort, and resulted in substantial overestimation of the expected number of deaths from CVD [23]. The sensitivity of the SCORE risk chart in predicting cardiovascular mortality in Finnish men and women has been shown to be $64 \%$ and $24 \%$, respectively [24]. High sensitivity means that the majority of the screened population might get a positive test result.

In conclusion, our results suggest that the SCORE risk chart does not take into account typical cardiovascular risk factors in women. Given the simplicity and suitability of ABI and eGFR measurements to daily clinical practice, and their potential ability to recognize high-risk individuals - especially women more systematic use of these measurements in the general practice seems justified.

\section{Acknowledgements}

The work was supported by the University of Turku, Turku, Finland.

\section{Declaration of interest}

The authors report no conflict of interest. The authors alone are responsible for the content and writing of the paper.

\section{References}

[1] European cardiovascular disease statistics 2008. Available at: http://www.ehnheart.org/files/statistics

[2] Conroy RM, Pyörälä K, Fitzgerald AP, Sans S, Menotti A, De Backer G, et al. on behalf of the SCORE project group. Estimation of ten year risk of fatal cardiovascular disease in Europe: The SCORE project. Eur Heart J 2003;24:987-1003.

[3] Stramba-Badiale M, Fox KM, Priori SG, Collins P, Daly C, Graham I, et al. Cardiovascular diseases in women: A statement from the policy conference of the European Society of Cardiology. Eur Heart J 2006;27:994-1005.

[4] Alberti KG, Zimmet P, Shaw J.; IDF Epidemiology Task Force Consensus Group. The metabolic syndrome: A new worldwide definition. Lancet 2005;366:1059-62.

[5] World Health Organization: Definition and diagnosis of diabetes mellitus and intermediate hyperglycemia. Report of a WHO/IDF consultation. Geneva: World Health Organization; 2006.

[6] Korhonen P, Aarnio P, Saaresranta T, Jaatinen P, Kantola I. Glucose homeostasis in hypertensive subjects. Hypertension 2008;51:945-9.

[7] Levey AS, Coresh J, Greene T, Marsh J, Stevens LA, Kusek J. Expressing the MDRD study equation for estimating GFR with IDMS traceable (gold standard) serum creatinine values. J Am Soc Nephrol 2005;16:69A.

[8] Espinola-Klein C, Rupprecht HJ, Bickel C, Lackner K, Savvidis S, Messow CM, et al. for the Atherogene Investigators. Different calculations of ankle brachial index and their impact on cardiovascular risk prediction. Circulation 2008;118:961-7.

[9] Task Force for the Management of Arterial Hypertension of the European Society of Hypertension (ESH) and of the European Society of Cardiology (ESC). 2007 Guidelines for the management of arterial hypertension. J Hypertens 2007;25: 1105-87.

[10] Daly CA, Clemens F, Sendon JL, Tavazzi L, Boersma E, Danchin N, et al. Euro Heart Survey Investigators. The clinical characteristics and investigations planned in patients with stable angina presenting to cardiologists in Europe: From the Euro Heart Survey of Stable Angina. Eur Heart J 2005;26:996-1010.

[11] Bennet L, Larsson C, Söderström M, Råstam L, Lindblad U. Diastolic dysfunction is associated with sedentary leisure time physical activity and smoking in females only. Scand J Prim Health Care 2010;28:172-8.

[12] Collins TC, Suarez-Almazor M, Bush LR, et al. Gender and peripheral arterial disease. J Am Board Fam Med 2006;19: 132-40.

[13] McDermott M, Greenland P, Liu K. Sex differences in peripheral arterial disease: Leg symptoms and physical functioning. J Am Geriatr Soc 2003;51:222-8.

[14] Fowkes FGR, Murray GD, Butcher I, Heald CL, Lee RJ, Chambless LE, et al. Ankle brachial index combined with Framingham risk score to predict cardiovascular events and mortality: A meta-analysis. JAMA 2008;300:197-208.

[15] Dormandy JA, Rutherford RB: Management of peripheral artery disease (PAD). TASC Working Group. Trans Atlantic Inter-Society Consensus (TASC). J Vasc Surg 2000;31:S1-44.

[16] Fowkes FGR, Housley E, Macintyre CCA, Prescott RJ, Ruckley CV.Variability of ankle and brachial systolic pressures 
in the measurement of atherosclerotic peripheral arterial disease. J Epidemiol Community Health 1988;42:128-33.

[17] Fowkes FGR, Low LP, Tuta S, ym. Ankle-brachial index and extent of atherothrombosis in 8891 patients with or at risk of vascular disease: Results of the international AGATHA study. Eur Heart J 2006;27:1861-7.

[18] Diehm C, Schuster A, Allenberg JR, Darius H, Haberl R, Lange $S$, et al. High prevalence of peripheral artery disease and co-morbidity in 6880 primary care patients: Crosssectional study. Atherosclerosis 2004;172:95-105.

[19] Zheng ZJ, Sharrett AR, Chambless LE, Rosamond WD, Nieto FJ, Sheps DS, et al. Associations of ankle-brachial index with clinical coronary heart disease, stroke and preclinical carotid and popliteal atherosclerosis: The atherosclerosis risk in communities (ARIC) study. Atherosclerosis 1997;131: $115-25$.

[20] Manjunath G, Tighiouart H, Ibrahim H, MacLeod B, Salem $\mathrm{DN}$, Griffith JL, et al. Level of kidney function as a risk factor for atherosclerotic cardiovascular outcomes in the community. J Am Coll Cardiol 2003;41:47-55.

[21] Ford ES, Zhao G, Li C. Pre-diabetes and the risk for cardiovascular disease. J Am Coll Cardiol 2010;55:1310-1317.

[22] Getz L, Sigurdsson JA, Hetlevik I, Kirkengen AL, Romundstad S, Holmen J. Estimating the high risk group for cardiovascular disease in the Norwegian HUNT 2 population according to the 2003 European guidelines: Modelling study. BMJ 2005;331:551-5.

[23] Stenlund H, Lönnberg G, Jenkins P, Norberg M, Persson M, Messner $\mathrm{T}$, et al. Fewer deaths from cardiovascular disease than expected from the Systematic Coronary Risk Evaluation chart in a Swedish population. Eur J Cardiovasc Prev Rehabil 2009; 16:321-4.

[24] Ketola E, Laatikainen T, Vartiainen E. Evaluating risk for cardiovascular diseases: Vain or value? How do different cardiovascular risk scores act in real life. Eur J Public Health 2010;20:107-12. 